\title{
CIENCIAMATRIA
}

Revista Interdisciplinaria de Humanidades, Educación, Ciencia y Tecnología

Año VIII. Vol. VIII. Nro 1. Edición Especial. 2022

Hecho el depósito de ley: pp201602FA4721

ISSN-L: 2542-3029; ISSN: 2610-802X

Universidad Nacional Experimental Francisco de Miranda (UNEFM). Santa Ana de Coro. Venezuela

Rously Eedyah Atencio-González; Alianny Madelyne Espinoza-Mendoza;

Anthony Nicodemo Zambrano-Morán

DOI $10.35381 / \mathrm{cm} . v 8 \mathrm{i} 1.659$

\section{Derecho humanitario en los adultos mayores}

\section{Humanitarian law for older adults}

Rously Eedyah Atencio-González

uq.rouslyatencio@uniandes.edu.ec

Universidad Regional Autónoma de los Andes, Quevedo

Ecuador

https://orcid.org/0000-0001-6845-1631

Alianny Madelyne Espinoza-Mendoza

dq.aliannymem49@uniandes.edu.ec

Universidad Regional Autónoma de los Andes, Quevedo

Ecuador

https://orcid.org/0000-0002-1123-1088

Anthony Nicodemo Zambrano-Morán

dq.anthonynzm49@uniandes.edu.ec

Universidad Regional Autónoma de los Andes, Quevedo

Ecuador

https://orcid.org/0000-0002-5404-7918

Recibido: 15 de septiembre 2021

Revisado: 10 de noviembre 2021

Aprobado: 15 de diciembre 2021

Publicado: 01 de enero de 2022 
CIENCIAMATRIA

Revista Interdisciplinaria de Humanidades, Educación, Ciencia y Tecnología

Año VIII. Vol. VIII. Nro 1. Edición Especial. 2022

Hecho el depósito de ley: pp201602FA4721

ISSN-L: 2542-3029; ISSN: 2610-802X

Universidad Nacional Experimental Francisco de Miranda (UNEFM). Santa Ana de Coro. Venezuela

Rously Eedyah Atencio-González; Alianny Madelyne Espinoza-Mendoza;

Anthony Nicodemo Zambrano-Morán

\section{Estimado Editor (a):}

Los adultos mayores representan un legado generacional en todas las culturas del mundo, en virtud de simbolizar el patrimonio de las familias y de la sociedad en general, que va en crecimiento, al respecto Montero Solano, Vega Chaves y Hernández (2017) manifiestan:

La población adulta mayor está en aumento rápido y progresivo a nivel mundial, lo cual asocia del mismo modo un incremento progresivo de abuso y maltrato en la vejez, incluso en formas silentes, con fuertes implicaciones en la calidad de vida de esta población. (p.1)

A pesar de constituir una población de gran valor en la sociedad, es en la mayoría de los casos maltratado, vulnerando sus derechos humanos establecidos en las normas internaciones y en las leyes de cada Nación o Estado, por lo cual Dabove (2020) indica lo siguiente:

En el Estado constitucional que rige en nuestra comunidad, el sistema republicano, la democracia y los derechos humanos son los criterios rectores para organizar la sociedad. Pero es bueno recordar también que todos ellos son válidos tanto en tiempos de normalidad, como de emergencia; en situaciones previsibles, tanto como en estados de excepción. (p.7)

En este tiempo de pandemia, se observa que los adultos mayores han sufrido grandes consecuencias que han afectado su salud y la vida de muchos de ellos se vio comprometida, llegando a cifras alarmantes de muertes en todo el mundo, lo que se traduce en políticas erradas en cuanto a la atención de este grupo vulnerable, lo que se relaciona con los planteamientos de Ruelas-González, Pelcastre-Villafuerte y ReyesMorales (2014):

La Organización Mundial de la Salud establece que en América Latina y el Caribe las deficiencias del sistema de atención para los AM, incluidas la mala capacitación del personal, la sobrecarga de trabajo, la mala atención y el deterioro de las instalaciones, hacen difícil la interacción entre el personal y los AM. (p.632) 
Lo indicado por la Organización Mundial de la Salud, relacionado a la atención de los adultos mayores (AM), constituye una violación de sus derechos fundamentales como lo es el derecho a la vida y la salud, los mismos constituyen factores de suma importancia en esta etapa de la vida de los seres humanos.

La vejez constituye un derecho, y en la cual los gobiernos mediante sus políticas públicas deben establecer los mecanismos, controles, planes para gestionar una atención integral y un buen vivir durante esta etapa de la vida, en este orden de ideas Dabove (2016) plantea que:

El Derecho de la Vejez, denominado también Derecho de la Ancianidad, es una nueva especialidad transversal destinada al estudio de la condición jurídica de las personas mayores, de 60 años de edad en adelante, en el derecho interno, regional e internacional. Este derecho se propone también el reconocimiento de las situaciones de aminoración, vulnerabilidad, discriminación, inestabilidad o abusos que puedan padecer estos sujetos, por el hecho de ser "viejos". (p.40)

El derecho humanitario en los adultos mayores implica el reconocimiento social, legal de este grupo de ciudadanos que forman parte de una sociedad en crecimiento, el ingreso de las personas mayores como sujeto de derechos debe implicar el reconocimiento de ciertos aspectos específicos como grupo de población, pero también debe ubicar un proceso de emancipación y lucha política (Palma, Perrotta y Rovira, 2019, p. 8)

En este sentido, la Comisión Económica para América Latina y el Caribe (2011), estableció mediante la convención Interamericana lo derechos de protección a los adultos mayores, de acuerdo a los siguientes artículos:

Artículo 1. Ámbito de aplicación y objeto El objeto de la Convención es promover, proteger y asegurar el reconocimiento y el pleno goce y ejercicio, en condiciones de igualdad, de todos los derechos humanos y libertades fundamentales de la persona mayor, a fin de contribuir a su plena inclusión, integración y participación en la sociedad.

Lo dispuesto en la presente Convención no se interpretará como una limitación a derechos o beneficios más amplios o adicionales que reconozcan el derecho 
internacional o las legislaciones internas de los Estados Parte, a favor de la persona mayor.

Artículo 2. Discriminación: Cualquier distinción, exclusión, restricción que tenga como objetivo o efecto anular o restringir el reconocimiento, goce o ejercicio en igualdad de condiciones de los derechos humanos y las libertades fundamentales en la esfera política, económica, social, cultural o en cualquier otra esfera de la vida pública y privada.

Discriminación múltiple: Cualquier distinción, exclusión o restricción hacia la persona mayor fundada en dos o más factores de discriminación.

Discriminación por edad en la vejez: Cualquier distinción, exclusión o restricción basada en la edad que tenga como objetivo o efecto anular o restringir el reconocimiento, goce o ejercicio en igualdad de condiciones de los derechos humanos y libertades fundamentales en la esfera política, económica, social, cultural o en cualquier otra esfera de la vida pública y privada.

Sin duda alguna los Estados del mundo, han tomado en serio el respeto por los derechos humanitarios de los adultos mayores, mediante la promoción de planes, leyes, normas y políticas públicas que les permitan un buen vivir; sin embargo, queda mucho por realizar en favor de las personas que han dado su vida por el desarrollo de la sociedad.

\section{FINANCIAMIENTO}

No monetario.

\section{AGRADECIMIENTO}

A la Universidad Regional Autónoma de los Andes, Quevedo; por motivar el desarrollo de la Investigación. 
CIENCIAMATRIA

Revista Interdisciplinaria de Humanidades, Educación, Ciencia y Tecnología

Año VIII. Vol. VIII. Nro 1. Edición Especial. 2022

Hecho el depósito de ley: pp201602FA4721

ISSN-L: 2542-3029; ISSN: 2610-802X

Universidad Nacional Experimental Francisco de Miranda (UNEFM). Santa Ana de Coro. Venezuela

Rously Eedyah Atencio-González; Alianny Madelyne Espinoza-Mendoza;

Anthony Nicodemo Zambrano-Morán

\section{REFERENCIAS CONSULTADAS}

Comisión Económica para América Latina y el Caribe (2011). Convención Interamericana sobre la protección de los derechos humanos de las personas mayores. [InterAmerican Convention on the Protection of the Human Rights of Older Persons] Recuperado de: https://n9.cl/szkd1

Dabove, M. I. (2016). Derechos humanos de las personas mayores en la nueva Convención Americana y sus implicancias bioéticas. [Human rights of the elderly in the new American Convention and its bioethical implications] Revista Latinoamericana de Bioética, 16(1), 38-59. DOI: http://dx.doi.org/10.18359/rlbi.1440.

Dabove, M.I. (2020). Derecho de la Vejez en tiempos de pandemia. [Old Age Law in Times of Pandemic] Revista de la Facultad de Derecho, (49), 1-20. https://dx.doi.org/10.22187/rfd2020n49a10

Montero-Solano, G., Vega-Chaves, J., y Hernández, G. (2017). Abuso y Maltrato en el Adulto Mayor. [Abuse and Maltreatment in the Elderly] Medicina Legal de Costa Rica, 34(1), 120-130. Recuperado de: https://n9.cl/j7fma

Palma, A., Perrotta, V., y Rovira, A. (2019) Las personas mayores como sujetos de derecho: el aporte de la convención Interamericana sobre la protección de los derechos de las personas mayores. [Older persons as subjects of law: the contribution of the Inter-American Convention on the Protection of the Rights of Older Persons] Recuperado de: https://n9.cl/orucd

Ruelas-González, M., Pelcastre-Villafuerte, B., y Reyes-Morales, H. (2014) Maltrato institucional hacia el adulto mayor: percepciones del prestador de servicios de salud y de los ancianos. [Institutional abuse of the elderly: perceptions of the health service provider and the elderly] Salud pública de México, 54, (6);631-637. Recuperado de: https://n9.cl/5ij02 